\title{
Horn Antenna Miniaturization with Integrated High Impedance Surfaces
}

\author{
Michael A. Forman \\ Sandia National Laboratories, Livermore, CA 94550
}

\section{Introduction}

With the miniaturization of wireless systems, antenna volumes are being reduced while output power remains constant. Novel horn antennas incorporating Sievenpiper high-impedance electromagnetic surfaces are shown in the following work to provide a reduction in volume. Functioning as TEM horn antennas, they operate at geometries that would otherwise be below the $\mathrm{TE}_{1,0}$ cutoff frequency and are capable of carrying more power than microstrip antennas with equivalent volumes. Four antennas in two geometries with metal and HIS sidewalls are designed, measured, and compared.

\section{High Impedance Surface}

A HIS is an electrically thin in-phase reflector, which provides surface-wave suppression. Within a given frequency band the currents from an adjacent antenna and its image are in phase, as opposed to being $180^{\circ}$ out of phase as typical with conductors. A Sievenpiper HIS is realized as an array of capacitive patches connected to a ground plane with vias [1]. The geometry and thus the lumped-circuit equivalents are tuned to exhibit high impedance over a predetermined frequency band. The structure is a composite right/left-handed (CRLH) metamaterial whose subwavelength unit cells act as a homogeneous effective dielectric.

Surface impedance is modeled as a parallel resonant circuit that is tuned to exhibit high impedance over a predetermined frequency band. Fringing electric fields between adjacent top patches are represented as a capacitance and magnetic fields in the dielectric generated by current through the vias and ground are represented as an inductance. A sheet impedance is defined to be equal to the impedance of this equivalent parallel resonant circuit, with a resonant frequency that marks the center of the HIS frequency bandgap.

The bandwidth of the HIS region is defined as the frequencies, where the radiation drops to half of its maximum value and occurs where the surface impedance is equal to the impedance of free space. The total bandwidth is approximately equal to the characteristic impedance of the surface divided by the impedance of free space. This is the bandwidth over which the reflection coefficient falls between $\pm 90^{\circ}$ and represents the maximum usable bandwidth for a parallel antenna over a resonant surface. A unit cell of the fabricated HIS is shown in Figure 1. It is $20.2 \mathrm{~mm}(\lambda / 6)$ square with a center frequency of $2.45 \mathrm{GHz}$ and a $12 \%$ bandwidth $[2,3]$.

\section{TEM Horn Antennas}

From electromagnetics it is known that the transverse dimension of a waveguide must be at least one half wavelength to satisfy the boundary conditions needed for the propagation of an electromagnetic wave. To miniaturize a rectangular waveguide, traditionally one would fill it with a dielectric, where the transverse dimension would decrease by the square root of 


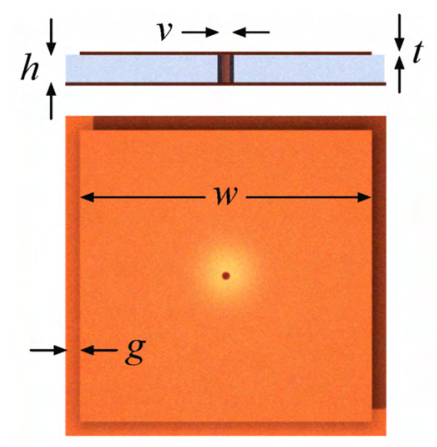

(a)

\begin{tabular}{c|l}
\hline Parameter & Value \\
\hline \hline Substrate & RT/Duroid 6006 \\
$e_{r}$ & 6.15 \\
$h$ & $2.54 \mathrm{~mm}$ \\
$t$ & $8.5 \mu \mathrm{m}$ \\
$w$ & $18.2 \mathrm{~mm}$ \\
$g$ & $1.0 \mathrm{~mm}$ \\
$v$ & $1.0 \mathrm{~mm}$ \\
\hline
\end{tabular}

(b)

Figure 1: Unit cell of the high-impedance surface (a) with design parameters (b).
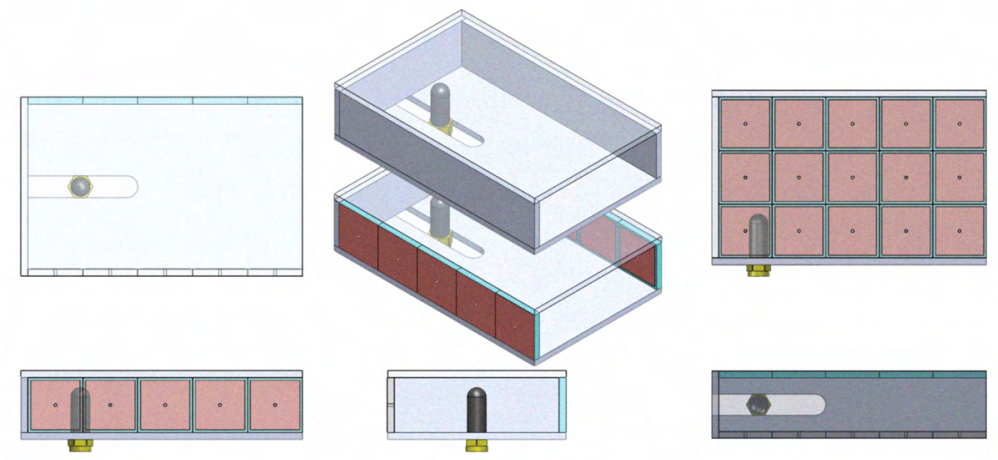

(a)

\begin{tabular}{c|c}
\hline H31 & H13 \\
\hline \hline$w=60.6 \mathrm{~mm}$ & $w=20.2 \mathrm{~mm}$ \\
$h=20.2 \mathrm{~mm}$ & $h=60.6 \mathrm{~mm}$ \\
$f_{c}=2.47 \mathrm{GHz}$ & $f_{c}=7.42 \mathrm{GHz}$ \\
\hline
\end{tabular}
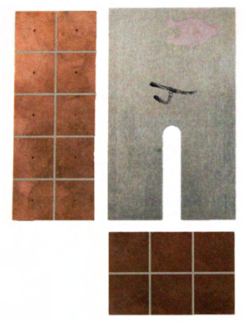

(c)

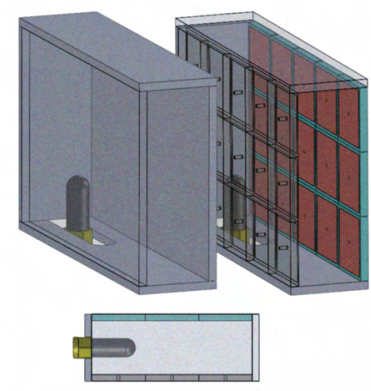

(b)
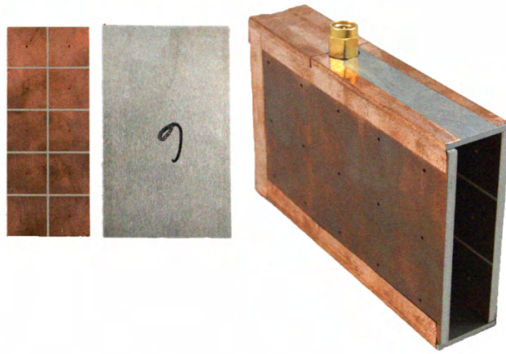

(d)

Figure 2: Four antennas in two geometries with metal and HIS sidewalls are designed. metal-walled $\mathrm{H} 31$ (a) and $\mathrm{H} 13$ (b) antennas have cutoff frequencies of $2.47 \mathrm{GHz}$ and $7.42 \mathrm{GHz}$ respectively (c). Antennas are modular (d) and tuned by moving a feed antenna in a channel.

the relative permittivity. Due to dielectric loss, difficulty in manufacturing, and maximum permittivities, this approach has limits.

As an alternative method, it has been shown that a TEM parallel-plate mode can be realized in a rectangular waveguide by replacing the metallic sidewalls with photonic bandgap structures $[4,5]$ and more recently with metamaterials [6]. Unlike a PBG material, a Sievenpiper HIS does not suppress surfaces waves through Bragg scattering from a periodic unit cell. 

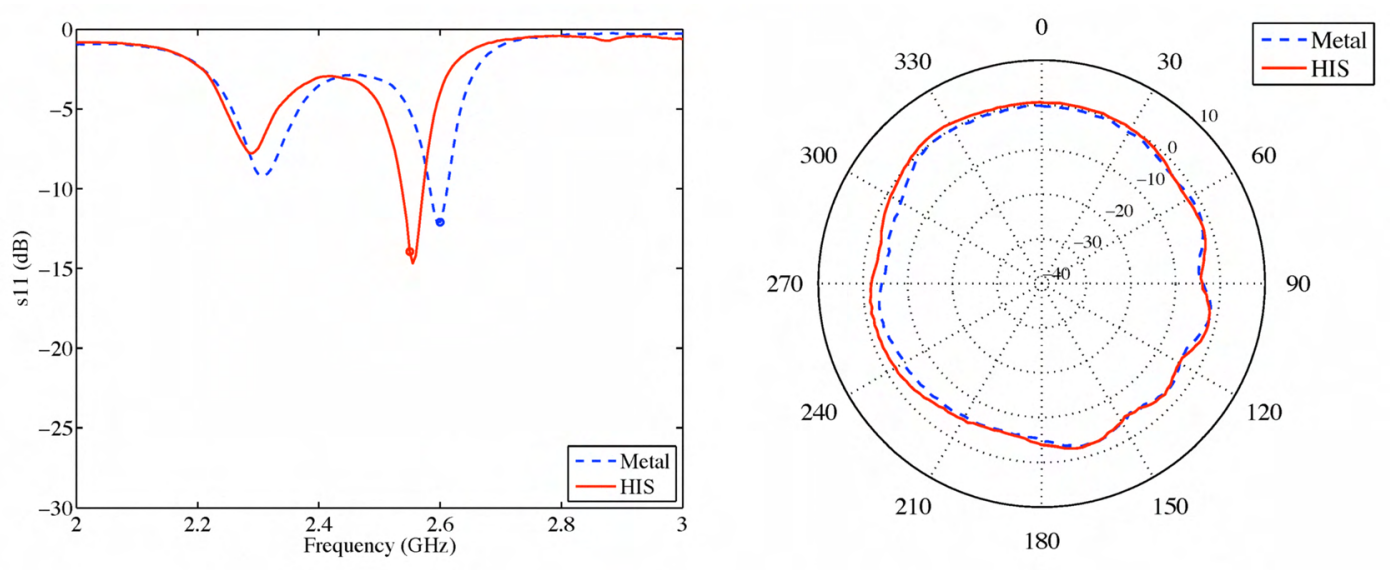

(a)
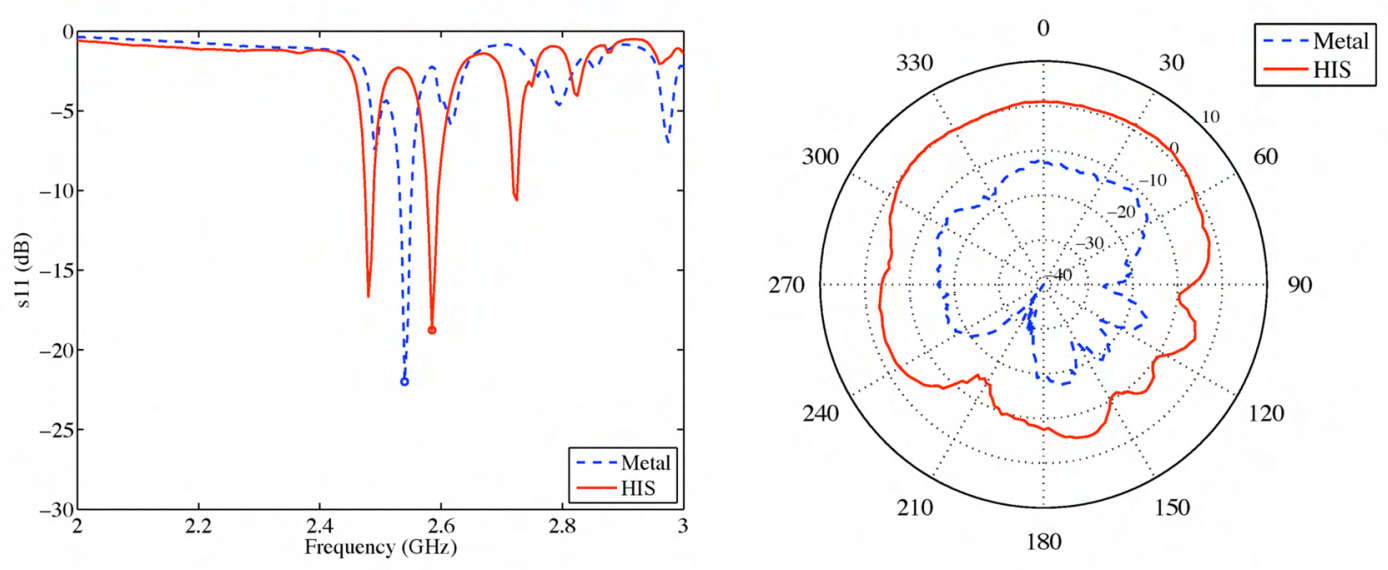

(b)

Figure 3: Measured insertion loss and E-plane far-field pattern measurements for the $\mathrm{H} 31$ (a) and H13 (b) antenna configurations. The H13 metal-walled antenna is in cutoff.

Thus a unit cell can be a fraction of the free-space wavelength, providing a greater potential for volume reduction. This work seeks to build upon this related work by implementing a reduced-volume rectangular horn antenna using the same principles but with a subwavelength Sievenpiper HIS.

Four antennas in two geometries with metal and HIS sidewalls are compared. Antenna dimensions are integer multiples of a HIS unit cell $(20.2 \mathrm{~mm})$ with a depth of five unit cells $(101.0 \mathrm{~mm})$. The first geometry, H31, shown in Figure 2(a) is three unit cells wide and one unit cell high. The metal- and HIS-walled H31 waveguides have simulated characteristic impedances of approximately $500 \Omega$ and $126 \Omega$ at $2.54 \mathrm{GHz}$ respectively. The second geometry, H13, shown in Figure 2(b) is one unit cell wide and three unit cells high. The metal-walled H13 waveguide is cutoff below $7.42 \mathrm{GHz}$. The HIS-walled H13 waveguide has a simulated characteristic impedance of $1130 \Omega$ at $2.54 \mathrm{GHz}$. The electric field is vertical in all antennas. Dimensions for all antennas and cutoff frequencies for metal-walled antennas are shown in Figure 2(c). 
The four horn antennas are assembled from modular pieces of machined aluminum and HIS, as seen in Figure 2(d). The top, bottom, and backs of the antennas are aluminum and the sides are either aluminum or HIS. The bottom aluminum plate has a channel to allow for the tuning of an inserted feed antenna. Because the characteristic impedance of each waveguide is different, the position of the feed antenna in the channel varies. An optimal match near $2.54 \mathrm{GHz}$ is found through tuning, the frequency sweep is performed, and the far-field pattern is measured.

The insertion loss and far-field radiation pattern of the H31 horns are shown in Figure 3(a). Pattern measurements are performed at the frequency indicated by the circle. The metalwalled $\mathrm{H} 31$ antenna is operating above its $\mathrm{TE}_{1,0}$ cutoff frequency of $2.47 \mathrm{GHz}$. As can be seen in the insertion-loss and far-field-pattern measurements, the metal- and HIS-walled antennas perform similarly. The insertion loss and far-field pattern of the H13 horns are shown in Figure 3(b). As can be seen in the far-field pattern measurements, the metalwalled antenna is operating below its $\mathrm{TE}_{1,0}$ cutoff frequency of $7.42 \mathrm{GHz}$, whereas the HIS-walled antenna is functioning in a TEM parallel-plate mode.

A horn antenna with an integrated Sievenpiper high-impedance surface has been shown to function at a frequency below the cutoff of a metallic horn antenna of the same dimension. Because the unit cell of a Sievenpiper HIS can be arbitrarily small, this antenna shows promise for further reductions in volume. In addition to reducing the size of the unit cell, future work will include improving bandwidth with a waveguide feed and comparing relative power-handling capability.

\section{References}

[1] D. Sievenpiper, L. Zhang, R. Jimenez Broas, N. Alexópolous, and E. Yablonovitch, "High-impedance electromagnetic surfaces with a forbidden frequency band," IEEE Transactions on Microwave Theory and Techniques, vol. 47, no. 11, 1999.

[2] J. R. Sohn, H. Tae, J. Lee, and J. Lee, "Comparative analysis of four types of highimpedance surfaces for low profile antenna applications," IEEE Antennas and Propagation Society International Symposium, vol. 1A, pp. 758-761, jul 2005.

[3] S. Clavijo, R. E. Diaz, and W. E. McKinzie III, "Design methodology for sievenpiper high-impedance surfaces: an artificial magnetic conductor for positive gain electrically small antennas," IEEE Transactions on Antennas and Propagation, vol. 51, pp. 26782690 , oct 2003.

[4] F. Yang, K. Ma, Y. Qian, and T. Itoh, "A novel tem-waveguide using uniplanar compact photonic band-gap (uc-pbg) structure," Asia Pacific Microwave Conference, vol. 2, pp. 323-326, 1999.

[5] J. A. Higgins, H. Xin, and A. Sailer, "Characteristics of Ka band waveguide using electromagnetic crystal sidewalls," IEEE MTT-S International Microwave Symposium Digest, vol. 2, pp. 1071-1074, jun 2002.

[6] S. Hrabar, J. Bartolic, and Z. Sipus, "Waveguide miniaturization using uniaxial negative permeability metamaterial," IEEE Transactions on Antennas and Propagation, vol. 53, pp. 110-119, jan 2005. 